\title{
Study of Common Urinary Problems of Females at a Government Hospital in Central India
}

\author{
Roma Pradhan ${ }^{1}$, Raj Kumar Mathur ${ }^{1}$, Poonam Mathur², \\ Fareed Khan', Akshay Gupta ${ }^{1}$, Mansi Chowhan ${ }^{1}$ \\ ${ }^{1}$ Department of Surgery, Mahatma Gandhi Memorial Medical College, Indore, India \\ ${ }^{2}$ Department of Obstetrics and Gynaecology, Mahatma Gandhi Memorial Medical College, Indore, India \\ Email: drromapradhan@yahoo.com
}

Received April 13, 2012; revised May 5, 2012; accepted May 17, 2012

\begin{abstract}
Aim: The aim of this study is to identify the common urinary problems in Indian females, their etiology and symtomatology. Material and Methods: This is a prospective epidemiological study of 703 female patients with urinary problems, who attended the surgery and gynecology OPD (outpatient department) in our institution which is a tertiary referral centre in central India. Result: 58\% of patients were middle age (31 - 55) yrs. Burning micturition was the most common urinary complain (607 patients) followed by increased frequency of micturition (309 patients). 68\% females took months or even years to take first consultation after appearance of their symptoms. $14 \%$ percent of patients suffered from urinary incontinence (stress, urge and mixed). $86 \%$ of women had education level below fifth standard. About 58\% of females gave birth to 3 or more than 3 issues. About 254 (36\%) patients had one or more unassisted delivery at home. Thirty eight percent of patients still remained undiagnosed. More than $50 \%$ of undiagnosed females lie in middle age group. 68\% of undiagnosed patients took consultation months or years after their symptoms first appeared. Irritative symptoms are the most common presenting complains of these undiagnosed patients followed by pain in abdomen. Around $60 \%$ of patients had history of either mental distress, domestic violence or any neuropschiatric disease. This study only gives us an idea about the urinary problems faced by females and their probable causative factors. Many more studies need to be done involving multidisciplinary approach in order to benefit this section of our society.
\end{abstract}

Keywords: Common Urinary Problems; Undiagnosed

\section{Introduction}

Urinary problems affect millions of Indian, and while some of these problems aren't a serious threat to overall health, they are distressing and can negatively affect one's quality of life. Many women are either too shy or ashamed to talk about their bladder problems to anyone, including their doctor.

So though the urinary problems are frustrating, embarrassing and affect quality of life, why is no consultation is sought for long time? The answer lies in shyness or taboo assoaciated with it, illiteracy, unawareness, lack of motivation, low socioeconomic status and lack of easily accessible medical care in rural and semi-urban areas of India.

The study is focused on urinary problems common in Indian females, but not notified, hence go unnoticed including the symptoms related to urogenital system.

Some problems, especially like incontinence, are considered as a normal phenomena with increasing age by some females. Prevalence of lower urinary tract symptoms including incontinence is higher in females than in males $[1,2]$. One reason is the anatomical difference; female pelvis has wider outlet which predisposes for subsequent pelvic floor weakness [3]. The second is physiological; with increasing age change in hormonal status and physiology of female uro-genital tract pre dispose to voiding dysfunctions [4]. Another utmost important and modifiable factor is the silent trauma to the pelvic supports- the utmost important structure for proper functioning of lower urinary tract, in the form of multiple vaginal deliveries (specially unattended home deliveries) and various obstetric and gynaecological surgeries (caesarean section, hysterectomy etc.) [1,2].

\section{Aims}

The aim of this study is to identify the common urinary problems of Indian females, their etiology, symptomatology.

\section{Materials and Methods}

This is a prospective epidemiological study of 703 fe- 
male patients with urinary problems, who attended the surgery and gynecology OPD in our institution, which is a tertiary referral centre in central India. Patients included were above 18 years of age. Patients with major co-morbidity like malignancies of the uro-genital tract (cervix, urinary bladder, uterus, vagina, ovary etc.) were excluded. This study is based on history and clinical examination of patients. Patients urinary complains were noted along with the duration of complain. Parity of patient was noted along with the mode of delivery and place of delivery. History of any chronic disease or any history of any previous pelvic surgery was also noted. Probable diagnosis was made clinically and with help of investigations. Patients were explained about their complaints, investigations, available treatments, and motivated to come for follow up for further management.

\section{Results}

$58 \%$ of patients were middle age (31 - 55) yrs (Table 1). Burning micturion was the most common urinary complain (607 patients) followed by increased frequency of micturition (309 patients) (Table 2) 68\% females took months or even years to take first consultation after appearance of their symptoms (Table 3).

$44 \%$ percent (Table 4 ) of patients were suffering from urinary tract infection which was confirmed by urine culture reports. E. coli was the most common organism causing urinary tract infection (66\%) in our study.

Only 14\% (Table 4) of patients suffered from urinary incontinence (stress, urge and mixed).

$86 \%$ of women had education level below fifth standard. About $58 \%$ of females gave birth to 3 or more than 3 issues. About 254 (36\%) patients had one or more unassisted delivery at home.

$38 \%$ (Table 4) of patients still remained undiagnosed. More than $50 \%$ of undiagnosed females lie in middle age group.

$68 \%$ of undiagnosed patients took consultation months or years after their symptoms first appeared. Irritative symptoms [burning micturition (92\%), urgency (42\%), increased frequency of micturition (41\%)] are the most common presenting complains of these undiagnosed patients followed by pain in abdomen. Around $60 \%$ of patients had history of either mental distress, domestic violence or any neuropschiatric disease (Table 5).

\section{Discussion}

This is a prospective study of 703 patients attending surgery and gynaecology OPD of M.Y hospital for any urinary complains. Maximum patients visiting our hospital are either from low socio economic group or from rural areas. Urban scenario differs from the rural scenario and hence the problems faced by them also differ.
Table 1. Age distribution.

\begin{tabular}{ccc}
\hline Age group & No. of patients & Percentage \\
\hline $18-30$ & 242 & 34.4 \\
$31-55$ & 406 & 57.7 \\
$>55$ & 55 & 7.8 \\
\hline
\end{tabular}

Table 2. Prevalence of urinary problems.

\begin{tabular}{cc}
\hline Symptoms & No. of patients \\
\hline Frequency & 309 \\
Burning micturition & 607 \\
Retention (acute or chronic) & 25 \\
Dysuria & 54 \\
Lower abd. pain & 375 \\
Haematuria & 25 \\
Urgency & 239 \\
Incomplete voiding & 94 \\
Constipation & 49 \\
Urge incontinence & 78 \\
Stress incontinence & 45 \\
\hline
\end{tabular}

Table 3. Duration after which first medical advice was taken.

\begin{tabular}{llll}
\hline Duration & Days & Months & Years \\
\hline No. of patients & $236(34 \%)$ & $298(42 \%)$ & $169(24 \%)$ \\
\hline
\end{tabular}

Table 4. Probable diagnosis of patients studied.

\begin{tabular}{ccc}
\hline Probable diagnosis & No. of patients & Percentage \\
\hline UTI & 309 & 44 \\
Incontinence & 97 & 14 \\
Pelvic floor prolapse & 32 & 5 \\
Undiagnosed & 269 & 38 \\
\hline
\end{tabular}

Table 5. H/O mental distress/domestic violence/neuropsychiatric illness in undiagnosed patients.

\begin{tabular}{cc}
\hline $\begin{array}{c}\text { H/O mental distress/domestic } \\
\text { violence/neuropsychiatric illness }\end{array}$ & No. of patients \\
\hline Present & 161 \\
Absent & 108 \\
\hline
\end{tabular}

According to Table 1 of age distribution of urinary complains $58 \%$ of patients were middle age (31 - 55) yrs. Females in the elder age group coming for urinary problems to our institute are far less as most females in that age group belong to rural areas and depend either on their husband or their son to accompany them to the hospital. They even depend on them for financial support.

Burning micturition was the most common urinary complain of females (607 patients) followed by increased 
frequency of micturition (309 patients). Constipation was present in about 49 patients. Studies in middle-aged women also support a high prevalence of constipation among patients suffering from urinary tract dysfunction. The only prospective study in constipated elderly with concomitant LUTS demonstrates that the medical relief of constipation also significantly improves LUTS [5].

$44 \%$ percent of patients were suffering from urinary tract infection, which was confirmed by urine culture reports. E. coli was the most common organism causing urinary tract infection (66\%) in our study. The most common organism causing UTI for all gender was E. coli and Klebsiella [6].

Only $14 \%$ of patients suffered from urinary incontinence (stress, urge and mixed). According to many studies prevalence of urinary incontinence ranged from 5\% to $72 \%$ among community-dwelling women [7-9]. Our results of urinary incontinence lie in the lower end of the range because in our country urine leak is considered an age related problem and consultation is not sought because of embarrassment. Also because of lack of awareness and low education level they probably believe that nothing much can be done about it.

The significantly lower rate of stress incontinence is probably not because of prevalence but because of the treatment seeking behavior and the society structure in India. Here women's lives are shaped by customs that are centuries old. Women greatly depend on their husbands, sons or fathers to seek any help for their medical problem. They tend to live with their problem, suffering every second of their life without even saying a word.

Urge incontinence was found to be more common in our study as compared to stress incontinence. Stress incontinence is more common than urge incontinence according to many studies $[9,10]$. This difference in finding is probably because of the structure of the society and the psychosocial problems faced by Indian women. It is known that there is a positive association between psychiatric and physical disorders. There are two possible mechanisms to explain this. Psychiatric disorders may present with physical symptoms or physical illness may have psychological consequences. It has been considered that urge incontinence can occur as a result of autonomic arousal associated with anxiety/neuroticism, and most research into psychiatric aspect of incontinence to date has concentrated on this aspect [11-13]. 18 patients of stress incontinence (55\%) had history of unassisted vaginal delivery and multi parity (3 or more).

$86 \%$ of women had education level below fifth standard. Our study shows extremely low level of education as most of our patients belong to the rural area. The lack of education is the root cause of all the health related problems. An uneducated person does not know about hygiene and this lack of knowledge increases the chances of various infections including urinary tract infections.

$58 \%$ of females gave birth to 3 or more than 3 issues. About 254 (36\%) patients had one or more unassisted delivery at home. Multi parity is one of the major social issues in India. Zeal to have a male child is mainly responsible for multi parity. $68 \%$ females took months or even years to take first consultation after the appearance of first symptoms. The reason for this delay was again shyness, illiteracy, unawareness, lack of motivation, low socioeconomic status of the patients and lack of easily accessible medical care. Studies on attendance at rural primary health centers reveal that more males than females are treated in almost all parts of the country. In the study that researched women who had incontinence and who delayed seeking help, it was reported that $74 \%$ of the women had waited for one year and $46 \%$ had waited for three years before seeking help [14].

$38 \%$ percent of patients still remained undiagnosed. They suffer from urinary symptoms and problems but with no positive findings. More than $50 \%$ of undiagnosed females lie in middle age group. $68 \%$ of undiagnosed patients took consultation months or years after their symptoms first appeared. $60 \%$ of patients had history of mental distress, domestic violence or any neuro-psychiatric disease. The most probable diagnosis of these 38\% patients can be explained either on the basis of urethral syndrome or painful bladder syndrome.

The urethral syndrome is defined as women who have symptoms resembling a lower urinary tract infection but whose objective findings are negative that is, no abnormal midstream urinalysis findings, no positive bacterial culture, and no obvious finding on a pelvic examination. $[15,16]$. The etiology of this urethral syndrome is obscure and has been attributed to an anxiety neurosis $[17,18]$ In this study irritative symptoms [burning micturition (92\%), urgency (42\%), increased frequency of micturition (41\%)] are the most common presenting complains of these undiagnosed patients followed by pain in abdomen. Urethral syndrome is thought to affect $20 \%-30 \%$ of all adult women and it is particularly seen in young women. The exact incidence of urethral syndrome is unknown because of a lack of consensus in diagnosis [19] Risk factors include grand multi parity, delivery without episiotomy and two or more abortions [20]. About 58\% of undiagnosed patient had 3 or more than 3 deliveries. Various hypothetical etiologies have been put forward [21]. According to one study the most striking finding was a significantly higher than normal maximum urethral closure pressure. Abnormal and low urinary flow rates, instability of the intra urethral pressure at rest, incomplete funneling of the bladder neck, and distal urethral narrowing during voiding constitute other typical urodynamic findings in the female urethral syndrome [22]. According to another study, there is strong evidence 
that the microscopic paraurethral glands connected to the distal third of the urethra in the pre vaginal space are homologous to the prostate [23].

The possible psychogenic cause that has been used as the basis for treatment with anxiolytic drugs for the past 20 years has been supported by various publications studying the response of such patients to personality tests (Minnesota Multiphasic Personality Inventory), [24] measurements of pelvic floor tension, [25] or external sphincter reactivity [26]. A psychological study sagely suggests that perhaps these were normal women responding to the stress of continuing symptoms and inadequate diagnosis and treatment [27]. As no definitive etiology has been established urethral syndrome is treated on "trial and error method".

Another syndrome which also can not be diagnosed easily is painful bladder syndrome (PBS). In 2002, the International Continence Society (ICS) published new recommendations and, notably, proposed that Interstitial cystitis should be renamed painful bladder syndrome (PBS) [28]. The ICS diagnosis of PBS is based on suprapubic pain related to bladder filling, day or nighttime frequency, and the absence of other obvious pathology [29]. Psychosocial association with painful bladder syndrome is shown by one more study which reported that the syndrome was more common in those who experienced abuse, who are worried about someone close to them and those who were having trouble paying for basics. This suggests that patients with painful bladder syndrome may be benefited from multifaceted approach of combining medical, psychological and cognitive treatment.

Undiagnosed urinary problems form a bulk of the problems womenfolk face at large because their causes perhaps lie more towards the emotional disposition and situation that the women find themselves in. The mental distress, domestic violence and general anxiety that women have to face all lead to this condition. Women occupy the inner recesses of the household, isolated from the men of their own families and other outsiders. Verbalizing problems and conflicts is rare, so it is very unlikely that men will know of the stresses or inner conflicts of the womenfolk. Also they feel shy to share their problems especially related to genito urinary tract even with their husbands leave about any other member of their family. The result is pent up mental stress. Lack of sharing of thoughts starts the vicious cycle of stress, which is difficult for her to break because of her present position in the society and her home.

\section{Conclusion}

The urinary problems are very frustrating to patients and not relieved easily, lead to either multi practitioner approach by the patients or they consider them to be normal phenomenon with increasing age and do not come for follow up. This bunch of society needs to be properly diagnosed, counseled, motivated and a combined effort of uro-gynaecologist, physiotherapist and psychologist should be provided in such a tertiary medical centre for better treatment of patients, to make them comfortable and improve their quality of life. Literacy level, social status, cultural background and accessibility to health care resources may also contribute to urinary problems therefore an improvement on these factors can lead to improvement in the quality of life of women. This study only gives us an idea about the urinary problems faced by females and their probable causative factors. Many more studies need to be done involving multidisciplinary approach in order to benefit this section of our society.

\section{REFERENCES}

[1] A. Wald, "MPH Hysterectomy: A Risk Factor for Urinary Incontinence?” Watch Women's Health, 2007.

[2] G. Rortveit, A. K. Daltveit, Y. S. Hannestad and S. Hunskaar, "The Norwegian EPINCONT Study, Urinary Incontinence after Vaginal Delivery or Cesarean Section,” New England Journal of Medicine, Vol. 348, No. 10, 2003, pp. 900-907. doi:10.1056/NEJMoa021788

[3] R. W. Soames, et al., "Skeletal System,” In: P. L. Williams, L. H. Bannister, M. M. Berry, et al., Eds., Gray’s Anatomy, 38th Edition, Churchill Livingstone, New York, 1995, pp. 425-736.

[4] L. V. Swithinbank and P. Abrams, "A Detailed Description, by Age, of Lower Urinary Tract Symptoms in a Group of Community-Dwelling Women,” BJU International, Vol. 85, No. 2, 2000, pp. 19-24.

[5] M. A. Averbeck, "Helmut Madersbacher Constipation and LUTS-How Do They Affect Each Other?” International Braz J Urol, Vol. 37, No. 1, 2011, pp. 16-28. doi:10.1590/S1677-55382011000100003

[6] J. Bahadin, S. S. Teo and S. Mathew, "Singhealth Polyclinics Bedok, Aetiology of Community-Acquired Urinary Tract Infection and Antimicrobial Susceptibility Patterns of Uropathogens Isolated," Singapore Medical Journal, Vol. 52, No. 6, 2011, pp. 415-420.

[7] J. W. Yarnell, G. J. Voyle, C. J. Richards and T. P. Stephenson, "The Prevalence and Severity of Urinary Incontinence in Women," Journal of Epidemiology and Community Health, Vol. 35, No. 1, 1981, pp. 71-74. doi:10.1136/jech.35.1.71

[8] K. Holst and P. D. Wilson, "The Prevalence of Urinary Incontinence and Reasons for Not Seeking Treatment,” NZ Medical Journal, Vol. 101, 1988, pp. 756-758.

[9] S. Hunskaar, K. Burgio, A. C. Diokno, et al., "Epidemiology and Natural History of Urinary Incontinence (UI)," 2nd Edition, Incontinence, Paris, 2002.

[10] Y. Hannestad, G. Rortveit, H. Sandvik and S. Hunskaar, "A Community Based Epidemiological Survey of Female Urinary Incontinence. The Norwegian EPINCONT Study,” Journal of Clinical Epidemiology, Vol. 53, No. 
11, 2000, pp. 1150-1157. doi:10.1016/S0895-4356(00)00232-8

[11] W. K. Frewen, "An Objective Assessment of the Unstable Bladder of Psychosomatic Origin,” British Journal of Urology, Vol. 50, No. 4, 1978, pp. 246-249. doi:10.1111/j.1464-410X.1978.tb02818.x

[12] R. J. Hafner, S. L. Stanton and J. Guy, "A Psychiatric Study of Women with Urgency and Urge Incontinence," British Journal of Urology, Vol. 49, 1977, pp. 211-214. doi:10.1111/j.1464-410X.1977.tb04106.X

[13] A. Crisp and J. Sutherst, "Psychological Factors in Women with Urinary Incontinence," Proceedings of the 13th Annual Meeting of the International Continence Society, Aachen, 1983, pp. 174-176.

[14] L. H. Koch, "Help-Seeking Behaviours of Women with Urinary Incontinence: An Integrative Literature Review," Journal of Midwifery \& Women's Health, Vol. 51, No. 6, 2006, pp. e39-e44. doi:10.1016/j.jmwh.2006.06.004

[15] G. J. A. Gallagher, J. Z. Montgomerie and J. D. K. North, "Acute Infections of the Urinary Tract and the Urethral Syndrome in General Practice,” British Medical Journal, Vol. 1, 1965, pp. 622-662. doi:10.1136/bmj.1.5435.622

[16] E. M. Messing, “Urethral Syndrome,” In: P. C. Walsh, R. F. Gittes, A. D. Perlmutter and T. A. Stamey, Eds., Campbell's Urology, WB Saunders, Philadelphia, 1986, pp. 1087-1091.

[17] L. A. Gray and W. B. Pingelton, "Pathological Lesion of Female Urethram,” The Journal of the American Medical Association, Vol. 162, 1956, p. 1361. doi:10.1001/jama.1956.02970320009003

[18] R. Zufall, "Treatment of the Urethral Syndrome in Women," The Journal of the American Medical Association, Vol. 184, No. 11, 1963, pp. 894-895. doi:10.1001/jama.1963.73700240019019a

[19] M. K. Terris, et al., "Urethral Syndrome,” eMedicine, May 2009.

[20] H. Gurel, S. A. Gurel and M. K. Atilla, "Urethral Syndrome and Associated Risk Factors Related to Obstetrics and Gynecology," European Journal of Obstetrics \&
Gynecology and Reproductive Biology, Vol. 83, No. 1, 1999, pp. 5-7. doi:10.1016/S0301-2115(98)00153-5

[21] H. J. Roberts, "Bubble Bath Cystitis,” The Journal of the American Medical Association, Vol. 201, No. 3, 1967. doi:10.1001/jama.1967.03130030077026

[22] G. A. Barbalias and E. M. Meares Jr., "Female Urethral Syndrome: Clinical and Urodynamic Perspectives," Urology, Vol. 23, No. 2, 1984, pp. 208-212. doi:10.1016/0090-4295(84)90024-4

[23] R. F. Gittes and R. M. Nakamura, "Female Urethral Syndrome. A Female Prostatitis?” Western Journal of Medicine, Vol. 164, No. 5, 1996, pp. 435-438.

[24] C. C. Cardon, D. Osborne and J. W. Segura, "Psychologic Characteristics of Patients with Female Urethral Syndrome," Journal of Clinical Psychology, Vol. 35, No. 2, 1979, pp. 312-313.

doi:10.1002/1097-4679(197904)35:2<312::AID-JCLP227 0350218>3.0.CO;2-1

[25] R. A. Schmidt and E. A. Tanagho, "Urethral Syndrome or Urinary Tract Infection?” Urology, Vol. 18, No. 4, 1981, pp. 424-427. doi:10.1016/0090-4295(81)90410-6

[26] W. E. Kaplan, C. F. Firlit and H. W. Schoenberg, “The Female Urethral Syndrome: External Sphincter Spasm as Etiology,” Journal of Urology, Vol. 124, No. 1, 1980, pp. 48-49.

[27] A. M. Bernstein and H. C. Phillips, "A Psychophysiological Evaluation of Female Urethral Syndrome: Evidence for a Muscular Abnormality,” Journal of Behavioral Medicine, Vol. 15, No. 3, 1992, pp. 299-312. doi:10.1007/BF00845358

[28] P. Abrams, et al., "Report from the Standardisation SubCommittee of the International Continence Society," American Journal of Obstetrics \& Gynecology, Vol. 187, No. 1, 2002, pp. 116-126. doi:10.1067/mob.2002.125704

[29] J. W. Warren, W. A. Meyer, P. Greenberg, et al., "Using the International Continence Society's Definition of Painful Bladder Syndrome,” Urology, Vol. 67, No. 6, 2006, pp. 1138-1142. doi:10.1016/j.urology.2006.01.086 